\title{
Comparison of various varying mechanism version for modified nodal array approach in foot plantar pressure measurement system
}

\begin{abstract}
In this paper, we constructed and compared four version of varying mechanism for modified Nodal Array Approach (NAA) in foot plantar pressure measurement system. In order to achieve NAA goals in having low circuit complexity while maintaining simple reading and solving algorithm, the varying mechanism part needs to be designed carefully by considering the factors such as low in sensor calculation error, component usage and complexity as well as algorithm size. 31 experiment conditions has been tested on these readout circuits with different varying mechanism where the results was presented, analyzed and discussed. A conclusion has been made where the Switch version has been selected to be the most suitable varying mechanism for the modified NAA readout circuit.
\end{abstract}

Keyword: Resistive readout circuit; Resistive sensor array; Foot plantar application 